\title{
Formation of wide binary stars from adjacent cores
}

\author{
Andrei Tokovinin ${ }^{\star}$ \\ Cerro Tololo Inter-American Observatory, Casilla 603, La Serena, Chile
}

Accepted XXX. Received YYY; in original form ZZZ

\begin{abstract}
Wide gravitationally bound pairs of stars can be formed from adjacent prestellar cores that happen to move slowly enough relative to each other. These binaries are remnants of the primordial clustering. It is shown that the expected fraction of wide bound pairs in low-density star formation regions can be larger than the fraction of wide pairs in the field. On the other hand, wide binaries do not form or survive in dense clusters. Recent works on the separation distribution of young binaries, summarized here, confirm these expectations. Alternative formation mechanisms of wide binaries such as cluster dissolution or unfolding of triple stars cannot explain the large observed fraction of young wide pairs and therefore are not dominant. The fact that more than a half of wide pairs contain subsystems matches the general multiplicity statistics and does not imply that hierarchical multiplicity and wide binaries are genetically related.
\end{abstract}

Key words: binaries: general; methods: statistical

\section{INTRODUCTION}

Stars form by fragmentation and collapse of molecular clouds. Dense cores of molecular gas are believed to be the intermediate stage in this process, with each core forming one or several stars and the core mass function being similar to the stellar initial mass function (Alves et al. 2007). Multiple stars form by fragmentation of cores and help to get rid of excessive angular momentum by storing it in the orbital motion (Reipurth et al. 2014).

The typical separation of binaries formed by a fragmenting core is related to its size (on the order of $10^{4} \mathrm{AU}$ ) and rotation and is somewhere between 10 and $1000 \mathrm{AU}$ (Sterzik et al. 2003), matching the simulations by Lomax et al. (2015). The smallest separation on the order of $10 \mathrm{AU}$ is set by the so-called opacity limit to fragmentation (e.g. Goodwin et al. 2007, Sect. 4.1). Newly formed binaries continue to accrete gas and at the same time migrate, evolving into tighter pairs or even merging (Figure 1).

Stellar pairs with the semimajor axis $a$ of $10^{4} \mathrm{AU}$ or wider are known to exist in the field (Makarov et al. 2008). As the typical size of the cores is similar, on the order of the Jeans length, such wide binaries cannot be formed by fragmentation of a single core. Special wide-binary formation mechanisms have been proposed, such as cluster dissolution (Kouwenhoven et al. 2010; Moeckel \& Bate 2010) or "unfolding" of more compact dynamically unstable triple systems (Reipurth \& Mikkola 2012).

I explore the hypothesis that the components of wide binaries originate from different cores. Owing to the natural clustering of the newly born stars, wide pairs of such stars are very frequent. Some of them happen to be bound accidentally. The estimated fraction of the wide bound pairs is larger than the fraction of wide

* E-mail: atokovinin@ctio.noao.edu

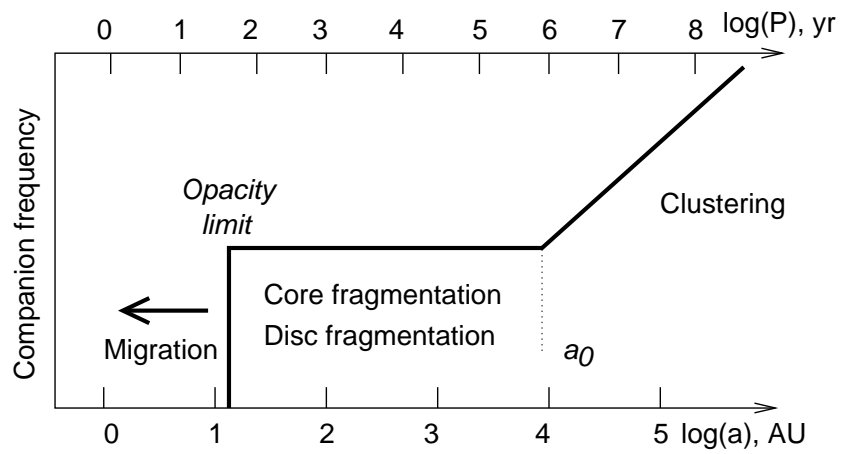

Figure 1. Characteristic scales of binary formation. The thick line shows schematically the number of stellar pairs vs. separation (on the lower axis) or period (on the upper axis). Fragmentation creates pairs with separations from 10 to $10^{4} \mathrm{AU}$, and some of those migrate to smaller separations. At the spatial scales larger than $10^{4} \mathrm{AU}$, the original fractal clustering of stars persists for a long time. The majority of very wide pairs in the clustering regime are not bound, but some are.

pairs in the field and even in the young stellar groups. Recently Joncour et al. (2017) discovered pairs of young stars in Taurus with separations up to $60 \mathrm{kAU}$ and argued that they are remnants of the original clustering.

The idea of wide binaries forming from two independent condensations has a long history. It was invoked by Abt \& Levy (1976) to explain the different mass ratio distributions in wide and close solar-type binaries. These distributions were revised by Duquennoy \& Mayor (1991), who nevertheless also found statistical differences between wide and close pairs and discussed "independent condensation" of wide binaries. More recent studies have 
shown that the mass ratio of solar-type binaries depends on the period weakly, if at all (Raghavan et al. 2010; Tokovinin 2014).

Duchêne \& Kraus (2013) discuss "extremely wide systems" in Section 5.5 of their review, defining them as binaries with separation 10 times wider than the (uncertain) empirical mass-dependent upper separation limit (about $10^{4} \mathrm{AU}$ for one solar mass). Another way to define wide binaries is by comparing the accretion time with the orbital period. If a protostar of one solar mass is assembled in $10^{5}$ years, the orbital period of a binary with $a>3 \mathrm{kAU}$ exceeds the star formation time.

Wide binaries move so slowly that it is extremely difficult or impossible to test observationally whether they are gravitationally bound or just have common spatial motion and distance (Proxima Cen is a good example, Kervella et al. 2017). Frequent occurrence of subsystems further complicates such tests. The term "wide pair" refers here to a pair of related, but not necessarily bound stars, reflecting this observational uncertainty. In contrast, "wide binary" means a bound system.

In Section 2, we show that the clustering and kinematics of young stars allows formation of a substantial number of wide bound binaries. Many of them contain closer subsystems because in a wide orbit there is a sufficient range of allowable stable periods around each component. The observed separation distribution and fractions of wide pairs are reviewed in Section 3; there are many such pairs in sparse environments, while in dense clusters there are few. Section 4 discusses alternative mechanisms of forming wide binaries that has been proposed in the literature. Section 5 is the summary.

\section{WIDE BINARIES AND CLUSTERING}

\subsection{The broken power law}

Larson (1995) discussed the distribution of separations between stars in the Taurus-Auriga star-forming region (SFR) by combining the study of the large-scale clustering by Gomez et al. (1993) with the statistics of close binary companions. He found a break in the power law that approximates the density of companions vs. angular separation $\theta$. At projected separations larger than $8250 \mathrm{AU}$, the density of companions per square degree follows the power law $\Sigma_{c}=3.4 \theta^{-0.62}$. This corresponds to the logarithmic separation distribution $f(\log s) \propto s^{1.38}$. Larson relates this to the fractal structure of the stellar clustering with a fractal dimension $D \approx 1.4$, inherited from the fractal structure of molecular clouds. On the other hand, at separations between 10 and $8250 \mathrm{AU}$ the surface density of companions in Taurus-Auriga is $\Sigma_{c}=0.0064 \theta^{-2.15}$, or $f(\log s) \propto s^{-0.15}$. The distribution of $\log s$ is almost flat and matches the Öpik's law for binaries. This range of (small) separations corresponds to binary stars. The total number of binary companions is about one per star.

Simon (1997) repeated the Larson's study and extended it to the Ophiuchus and Orion Nebular Cluster (ONC) SFRs. In all SFRs the companion statistics is well represented by the broken power law. The break point in the ONC is found at smaller separation around $400 \mathrm{AU}$. Simon argues that the transition between the clustering and binary regimes occurs at separation on the order of average projected distance between the stars, i.e. at the confusion limit. Bate et al. (1998) confirm this by simulations and note that the power law at large scales can have multiple origins and does not necessarily mean fractal clustering. For a sparse SFR like Taurus-Auriga, they find that the break in the power law indeed cor-

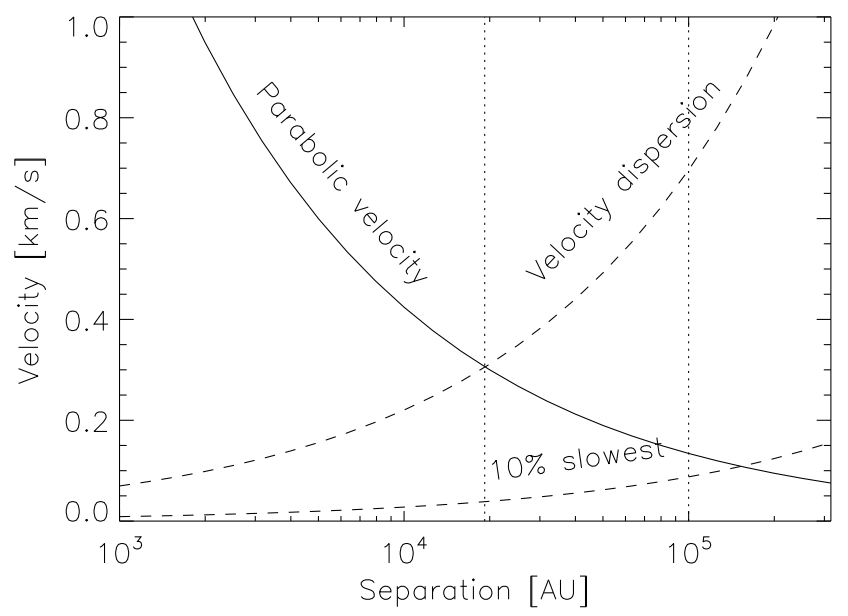

Figure 2. Dependence of the parabolic velocity for a solar-mass binary on its separation and the velocity dispersion in the molecular clouds. The lower curve shows the velocity of the $10 \%$ slowest stars, assuming normal distribution. At separation of $10^{5} \mathrm{AU}(0.5 \mathrm{pc})$, the dispersion is 5 times larger than the parabolic velocity, but about $15 \%$ of star pairs still can be bound if the relative velocity of the cores is normally distributed.

responds to the average spatial separation between the stars which is on the order of the Jeans length.

However, the transition between the clustering and binary regimes is smooth, stars formed in adjacent cores can "fall" to a common centre, interact dynamically, and produce a bound binary if their relative velocity is small enough. We estimate below the fraction of potentially bound wide pairs. Kraus \& Hillenbrand (2008) suggested that the binary and clustering regimes are separated by a small region with a constant density of companions where the primordial clustering has been destroyed by motions of the stars. Joncour et al. (2017) explored statistics of wide pairs in Taurus by several alternative methods and found a population of wide pairs with an approximately log-flat separation distribution extending to $60 \mathrm{kAU}$, with preferentially coeval components.

\subsection{Kinematics of cores and binaries}

Stars form in groups. The spatial locations of the newly formed stars and their velocities are inherited from the parent molecular cloud. Recent studies have shown that the gas is organized in filamentary structures. The diameters of the filaments are on the order of $0.1 \mathrm{pc}$, with a remarkably small dispersion around this typical value (André et al. 2014). Prestellar cores form along the filaments in chain-like linear configurations, with typical separations on the order of the filament diameter. Linear configurations correspond to the fractal dimension $D \sim 1$. The number of stars within a distance $R$ grows approximately as $R^{D}$. Larson (1995) found $D \approx 1.4$ for the Taurus-Auriga SFR, later Kraus \& Hillenbrand (2008) revised it to $D \approx 1$.

Gas motions in molecular clouds are described by the Larson's law, where the typical rms velocity difference between two fragments $\sigma_{V}$ is roughly proportional to the square root of their separation $s, \sigma_{V}(s) \approx(s / 1 \mathrm{pc})^{1 / 2}\left[\mathrm{~km} \mathrm{~s}^{-1}\right]$ (Heyer et al. 2009). On the other hand, the orbital velocity of a binary decreases with its semimajor axis $a$ as $V_{\text {orb }}=30 a^{-1 / 2} M^{1 / 2}\left[\mathrm{~km} \mathrm{~s}^{-1}\right], M$ being the mass sum in solar units and $a-$ semimajor axis in AU. This formula describes the differential velocity in a circular orbit, while the 
parabolic velocity $V_{\text {par }}=\sqrt{2} V_{\text {orb }}$. A pair of stars moving faster than $V_{\text {par }}$ has positive total energy and is not gravitationally bound.

Suppose that two gas fragments of a collapsing cloud form a binary with $a \approx s$, thus storing the angular momentum in its orbit. Then the condition $\sigma_{V} \leq V_{\mathrm{par}}$ must hold, otherwise the fragments will not be gravitationally bound to each other (Figure 2). This leads to

$$
s<a_{0}=1.910^{4} M^{1 / 2}[\mathrm{AU}],
$$

or $a_{0} \approx 0.1 \mathrm{pc}$. The maximum separation of a solar-mass binary $a_{0}$ is therefore defined by the gas motions to be on the order of $10^{4} \mathrm{AU}$. This is also the characteristic size of the prestellar cores and of the same order as the Jeans length and the typical diameter of filaments in molecular clouds. Note that this scale depends on mass; it is smaller for low-mass cores and larger for massive cores. Environments with faster gas motion should produce, on average, closer binaries.

\subsection{Fraction of wide bound pairs}

Cores separated by the distance $s>a_{0}$ on average move too fast to form a gravitationally bound pair. However, the motions are chaotic and a certain fraction of adjacent cores still happen to be bound.

Suppose that the relative velocity of cores is distributed normally with a dispersion $\sigma_{V}(s) \propto s^{1 / 2}$. The fraction of cores with a relative velocity less than $V$ is then approximately $\sqrt{2 / \pi} V / \sigma_{V}=$ $0.8 V / \sigma_{V}$, assuming $V \ll \sigma_{V}$. For bound pairs, we require $V<$ $V_{\text {par }}$, so the fraction of bound core pairs among all neighbors should decrease with separation as $0.8 V_{\mathrm{par}} / \sigma_{V} \propto s^{-1}$.

The initial clustering of young stars is such that the companion density is $f(s) \propto s^{0.4}$ (assuming $D=1.4$ ). The expected fraction of bound pairs with $s \gg a_{0}$ is therefore $f_{\text {bound }}(s) \propto s^{-0.6}$. The $\log$-flat distribution of separations corresponds to $f(s) \propto s^{-1}$, so the above argument predicts the number of wide bound pairs in excess of the Öpik's law, or matching it for $D \approx 1$.

If the relative motions of the cores are approximately isotropic, the modulus of the relative velocity $V$ is distributed according to the Maxwell-Bolzmann law

$$
f_{\text {Maxwell }}(V)=\sqrt{2 / \pi} V^{2} \sigma^{-3} \exp \left(-V^{2} / 2 \sigma^{2}\right) .
$$

In such case, the fraction of slow movers (bound pairs) decreases with $s$ much faster, as $\left(V / \sigma_{V}\right)^{3} \propto s^{-3}$. Therefore, in the case of isotropic relative motions the number of wide binaries than can be formed from adjacent cores drops sharply at $s>a_{0}$.

It is conceivable that in the filamentary clouds the relative motions are highly anisotropic, with one prevailing direction (e.g. quasi-rotation). If motions in one direction dominate, the Gaussian distribution of the relative velocity is a fair approximation, and the number of wide bound pairs can be significant. Bound wide binaries can also originate from regions with a slower than average relative gas motion. Decreasing the velocity dispersion by a factor of two doubles the $a_{0}$, extending it to $40 \mathrm{kAU}$.

\subsection{Time scale}

The orbital period of a binary is related to its semimajor axis by the third Kepler law, $P=a^{2 / 3} M^{-1 / 2}$, where $P$ is in years, $a$ is in $\mathrm{AU}$ and $M$ is the mass sum in solar units. The upper scale in Figure 1 shows the period vs. separation for a solar-mass binary. The characteristic scale $a_{0}=10^{4}$ AU corresponds to $P=1 \mathrm{Myr}$. The time needed for the two cores to fall to the centre of mass or to "unfold" is about $P / 2$. If the star formation and accretion last for about $10^{6}$ years, the pair of cores separated further than $a_{0}$ has not yet had time to fall onto each other and cannot be considered as a binary, even if they will eventually become bound.

The Larson's law predicts faster motions at larger separations; e.g., two stars separated by $1 \mathrm{pc}$ move at $\sim 1 \mathrm{~km} \mathrm{~s}^{-1}$ speed and will travel $1 \mathrm{pc}$ in $1 \mathrm{Myr}$. This primordial motion is not generally directed toward the centre of mass and most likely will move the stars apart. The $\sigma_{V}(s) \propto s^{1 / 2}$ dependence means that larger initial configurations will be preserved longer; the clustering at the spatial scale $s$ will persist for $t \sim(s / 1 \mathrm{pc})^{1 / 2}$ Myrs. In other words, the smallest spatial scale of the remnant clustering is proportional to $t^{2}$. Young objects of Class 0 have ages on the order of $10^{5}$ years and keep their primordial clustering at the scale of $0.01 \mathrm{pc}$ or $2 \mathrm{kAU}$, while at $10 \mathrm{Myr}$ this scale becomes $100 \mathrm{pc}$ and all clustering is lost. Kraus \& Hillenbrand (2008) demonstrate how the original clustering has been destroyed on small scales by modeling the observed companion density with a doubly broken power law. The knee positions in these fits match the velocity dispersion times age. A transition zone between the two power-law segments was also found for young binaries in the Orion molecular clouds by Kounkel et al. (2016).

By similar argument, tight pairs should form and evolve faster than the wide ones. The free-fall time is proportional to $\rho^{-1 / 2}$, so dense regions collapse in a runaway manner, creating singularities. However, those star embryos have only a small mass and acquire most of their final mass by accreting the surrounding gas. Larson (2007) notes that "self-gravitating structures may generally be built or organized from the bottom up because gravitational processes operate faster in smaller and denser regions, so that the matter is collected together first on small scales and then on progressively larger scales". This means that multiple stars form mostly "from inside out", starting with the inner subsystems and adding outer components. When the two cores combine in a bound wide binary, some of them already contain subsystems. We expect the orbits of those subsystems to be randomly aligned relative to each other and to the outer orbit.

\subsection{Why wide pairs often contain subsystems}

It has been noted that many wide pairs contain inner subsystems, i.e. are hierarchical triple, quadruple or higher-order multiples. For example, Elliott \& Bayo (2016) found that in the $\beta$ Pictoris moving group (BPMG), 11 out of of 14 pairs with projected separations larger than $10^{3} \mathrm{AU}$ are hierarchical multiples. Law et al. (2010) found that $45 \%$ of wide M-dwarf pairs are hierarchical multiples, with the fraction of hierarchies being larger at wider separations. Joncour et al. (2017) estimate that $\sim 68 \%$ of wide pairs in Taurus with $s>1 \mathrm{kAU}$ contain subsystems. High incidence of hierarchical systems with wide outer separations has been put forward as an argument for preferential formation of wide binaries by unfolding of more compact triples (Reipurth \& Mikkola 2012).

The statistics of solar-mass hierarchical multiple stars in the field can be modeled as an almost independent combination of the inner and outer subsystems drawn from the same generating distribution of periods and restricted only by the dynamical stability (Tokovinin 2014). In a wide pair, the range of dynamically stable inner separations is large, hence the incidence of subsystems increases with the outer separation. Suppose that this range corresponds to the subsystem probability $\epsilon=0.5$. The fraction of wide pairs without subsystems is then $(1-\epsilon)^{2}=0.25$, the remaining 
Table 1. Distribution of projected separations

\begin{tabular}{lcccc}
\hline $\log s(\mathrm{AU})$ & Perseus $^{a}$ & Taurus $^{b}$ & BPMG $^{c}$ & Simulations $^{d}$ \\
\hline$(-0.5,0)$ & $\ldots$ & $\ldots$ & 5 & 7 \\
$(0,0.5)$ & $\ldots$ & $\ldots$ & 4 & 23 \\
$(0.5,1)$ & $\ldots$ & 18 & 5 & 35 \\
$(1,1.5)$ & 3 & 17 & 6 & 44 \\
$(1.5,2)$ & 8 & 24 & 5 & 22 \\
$(2,2.5)$ & 5 & 12 & 3 & 24 \\
$(2.5,3)$ & 5 & 18 & 4 & 11 \\
$(3,3.5)$ & 9 & 10 & 7 & 2 \\
$(3.5,4)$ & 13 & 13 & 0 & 5 \\
$(4,4.5)$ & $\ldots$ & 23 & 4 & 2 \\
$(4.5,5)$ & $\ldots$ & 93 & 3 & 0 \\
$N_{\text {tot }}$ & 52 & 118 & 49 & 426 \\
\hline
\end{tabular}

References: ${ }^{a}$ Tobin et al. (2016); ${ }^{b}$ Joncour et al. (2017); ${ }^{c}$ Elliott \& Bayo (2016); ${ }^{d}$ Bate (2014).

$75 \%$ of wide pairs are hierarchical multiples. This corresponds to 10.5 hierarchies among the 14 wide pairs in the BPMG.

The field multiplicity model (Tokovinin 2014) does predict a mild correlation between the wide (outer) and close (inner) subsystems by postulating that the field is a mixture of binary-rich and binary-poor populations. Wide binaries come from the lowdensity SFRs that also have an increased binary fraction, hence a larger fraction of subsystems. Moreover, both Law et al. (2010) and Joncour et al. (2017) note that wide pairs containing subsystems have larger masses and larger binding energies compared to pure wide binaries, helping them to survive. The environment effect and the binding-energy argument may be sufficient for explaining the fraction and period distribution of hierarchies in the field without assuming special formation mechanisms of wide binaries related to hierarchical multiplicity.

\section{OBSERVED FREQUENCY OF WIDE PAIRS}

\subsection{Low-density SFRs}

Binarity of very young protostars in the Perseus SFR has been recently explored by Tobin et al. (2016) using high-resolution observations at centimeter wavelengths. Their resolution limit corresponds to the projected separation $s \sim 15$ AU. The distribution of $s$ for Class 0 and Class I sources from their Tables 3 and 4 is reproduced here in Table 1 as the number of pairs per 0.5 dex separation bin. The last line of this Table gives the total size of the sample, allowing us to compute the companion frequency. The age of all sources is less than $0.5 \mathrm{Myr}$, the majority of the Class 0 sources are younger than $\sim 160 \mathrm{Myr}$.

Table 1 also gives the latest binary statistics in Taurus based on the Table C1 from Joncour et al. (2017). I selected from their catalogue 142 stars with mass above $0.3 \mathcal{M}_{\odot}$ observed with high angular resolution, and computed the number of pairs in each separation bin, counting each pair only once. To match the common convention, 24 pairs of catalog entries within $10 \mathrm{kAU}$ of each other are considered as binary systems, reducing the sample size to 118 (this adjustment affects only the normalization of the curve). The full broken line without error bars in Figure 3 is a good match to the broken power law of Simon (1997). Kraus et al. (2011) also studied the separation distribution in Taurus, but only out to $5 \mathrm{kAU}$, avoiding the clustering regime.

Young moving groups have ages between 10 and $100 \mathrm{Myr}$;

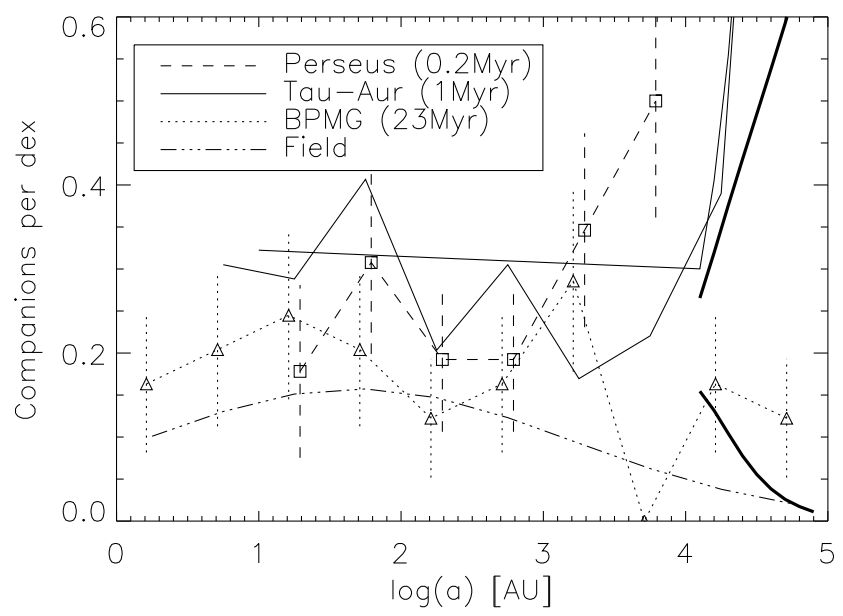

Figure 3. Distributions of projected separations in the Perseus SFR (dashed line, Tobin et al. 2016), Taurus-Auriga (two-segment and broken full lines, Simon 1997; Joncour et al. 2017), BPMG (dotted line, Elliott \& Bayo 2016), and the field (dash-dot line, Raghavan et al. 2010). Companion frequency per decade of separation is plotted on the vertical axis. The thick solid lines at $\log a>4$ are the clustering distributions scaled down according to the expected proportion of wide bound pairs under the assumptions of Gaussian (upper) or Maxwell (lower) velocity distributions.

the age of the $\beta$ Pictoris moving group (BPMG) is $23 \mathrm{Myr}$ (Mamajek \& Bell 2014). Elliott \& Bayo (2016) studied the binary statistics in this group using a variety of techniques. The sample size is 49 , the companion frequency is about one. Data from their Table 2 are used for computing the histogram given in the fourth column of Table 1. Kraus \& Hillenbrand (2009) derived a similar logarithmically flat separation distributions in the range $5<s<5000$ AU for binaries in both Taurus-Auriga and Upper Scorpius SFRs.

Binary statistics of the older field stars of approximately one solar mass is well studied (Raghavan et al. 2010; Tokovinin 2014). The distribution of periods and separations is approximately lognormal, with the median period of $10^{5}$ days, logarithmic period dispersion of 2.3, and companion fraction about 0.60. At $s>3 \mathrm{kAU}$, the frequency of companions declines approximately as a power law $f(s) \propto s^{-1.5}$ (Tokovinin \& Lépine 2012); the power law fits the data as well as the log-normal model. About $2 \%$ of solar-type stars in the field have companions with $s>10^{4}$ AU. Figure 3 plots the fraction of companions per decade of separation. The field distribution is shown by the dash-dot line, while the full line is the broken power law for Taurus-Auriga (data from Simon 1997). The clustering power law scaled down according to the expected fraction of bound pairs (thick solid lines) shows the frequency of potentially bound wide pairs originating from adjacent cores under two assumptions (normal or Maxwell distributions of the relative velocity).

In the youngest Perseus SFR there are many pairs with separations of $\sim 10^{3} \mathrm{AU}$ and wider, while at smaller separations the numbers are approximately constant, within the errors (a log-uniform distribution). A similar upturn in the separation distribution of protostars at $s>10^{3.5}$ AU has been found earlier by Conneley et al. (2008). In the older Taurus-Auriga SFR, the upturn occurs at separations $s>10^{4} \mathrm{AU}$, while in the BPMG the distribution is approximately flat out to $10^{5} \mathrm{AU}$ (note however the large statistical error bars).

The multiplicity fraction depends on the mass of the primary 


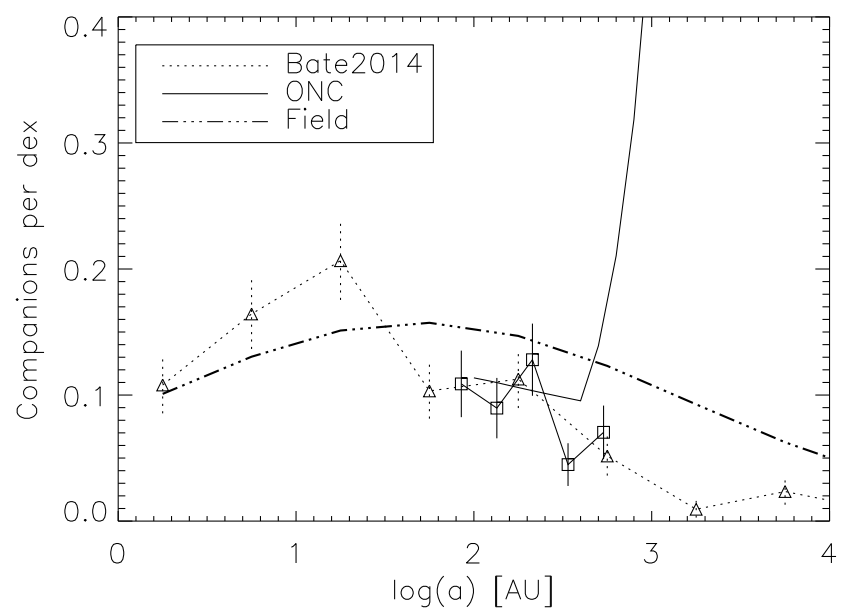

Figure 4. Distributions of projected separations in the ONC (full lines), in a simulated dense cluster (dotted line), and in the field.

component (Duchêne \& Kraus 2013). Data on the young associations plotted in Figure 3 refer to a mixture of masses, mostly below one solar, while the masses in Perseus are not known and these protostars are still accreting. The comparison of the multiplicity fraction with the field (masses around one solar) should take this into account. The histograms show that the multiplicity fractions in Taurus, BPMG and Perseus are larger than in the field even at moderate separations $s \sim 10^{2} \mathrm{AU}$, as in other sparse SFRs (Kraus \& Hillenbrand 2009; King et al. 2012). Such binaries are not disrupted dynamically and survive in the field. At the same time, sparse SFRs produce most (or all) wide binaries. Not surprisingly, those wide binaries often contain subsystems.

\subsection{Dense SFRs}

In dense regions, wide binaries cannot form or survive, owing to dynamical interactions with their neighbors. The typical distance between stars is less than the core size or the Jeans length, so the concept of core becomes questionable in these conditions. Protostars move in a common gas cloud while accreting and interacting dynamically. Bate (2014, and references therein) made large hydrodynamical simulations of the collapse of a dense turbulent cloud containing $500 M_{\odot}$ of gas. Simulations cover $0.3 \mathrm{Myr}$, until a substantial fraction of the gas is accreted. Although the filamentary structure and fractal-like clustering of newly formed stars develop initially, the subgroups subsequently merge, with violent dynamical interactions between the stars and continuing accretion. Citing Moeckel \& Bate (2010), "..the stars form in a structured fashion, with smaller sub-clusters merging to form a final cluster consisting of a tightly bound core with radius $\approx 0.05$ pc surrounded by an expanding halo of ejected stars." We provide in Table 1 the distribution of the semimajor axes from (Bate 2014) that drops sharply at $a>1 \mathrm{kAU}$. Subsequent dynamical evolution of the cluster may curtail this distribution even further (Kaczmarek et al. 2011). However, Moeckel \& Bate (2010) argue that after the gas dispersal the cluster expands and does not destroy the binaries that have already formed in a denser environment. The multiplicity fraction in the expanding cluster stays approximately constant, except in its outer halo where it is $\sim 2$ times less.

Figure 4 plots the separation distribution for the high-density SFR, namely the ONC. Squares and full line correspond to the Figure 7 of Reipurth et al. (2007), who studied the binarity in the sep- aration range from 67.5 to $675 \mathrm{AU}$ in a sample of 781 stars outside the Orion Trapezium. The broken power law is based on the Simon (1997) fit to the companion statistics in the Trapezium. It is reduced by a factor 2 to match approximately the binary fraction found by Reipurth et al. Such adjustment is justified by the large errors of the power-law fits in (Simon 1997) and by the larger primary mass (hence higher multiplicity) in the Trapezium sample. Bate et al. (1998) convincingly show that the break point in the power-law fits is related to the average star density, which is high in the Trapezium. We do not see the sharp upturn in the separation distribution of binaries outside the Trapezium because the stellar density there is much less, and because the contribution of neighbouring stars has been subtracted from the histogram.

For comparison, we plot in Figure 4 the separation distribution of binaries resulting from the large hydro-dynamical simulations by Bate (2014) (see Table 1). The simulated cluster roughly matches the ONC in density. Note the sharp drop of binary frequency at separations beyond $300 \mathrm{AU}$. There are still a few soft binaries with $s>10^{3} \mathrm{AU}$ that will likely be disrupted within the cluster, while new wide binaries will appear in its expanding halo (see Section 4.2).

At separations around $100 \mathrm{AU}$, the companion frequency in the ONC and in the simulated cluster is less than for the solar-field dwarfs. This statistics refer to primary stars of less than one solar mass, hence such difference is expected. Of importance here is the fast drop in the number of pairs wider than $\sim 300 \mathrm{AU}$, compared to the separation distribution in the field and in the low-density SFRs. Reipurth et al. (2007) further discuss the paucity of wide pairs in the ONC.

\section{ALTERNATIVE FORMATION MECHANISMS OF WIDE BINARIES}

\subsection{Unfolding}

Reipurth \& Mikkola (2012) suggested that very wide binaries are formed by ejections from more compact unstable triples. They call this "unfolding" of triple systems into extreme hierarchical architecture. In this mechanism, the outer orbits are very eccentric because the angular momentum of the wide pair is derived from the initially more compact system. Although the ratio of semimajor axes in unfolded triples can be large, they are still only marginally stable because the tertiary and the binary approach each other at the periastron of the eccentric outer orbit.

The unfolding time is on the order of $P / 2$. The authors note that "Many wide systems have not unfolded fully at $1 \mathrm{Myr}$, and the most extreme wide systems will take tens to hundreds of million years to unfold, and they are thus more protected against disruption by passing stars." In young associations such as TaurusAuriga (age $\sim 1 \mathrm{Myr}$ ), there should be no binaries with $s>10^{4} \mathrm{AU}$ formed by this mechanism. In reality, young SFRs contain many wide pairs (including unbound ones), but their fraction progressively decreases with age instead of increasing.

In the simulations by Reipurth \& Mikkola (2012), decaying triple systems generate about $2 \%$ of wide $\left(a>10^{4} \mathrm{AU}\right)$ binaries per system, matching approximately the frequency of wide pairs in the field. However, not all cores fragment into triple or quadruple stars, and not all triples are unstable, so in a realistic situation the frequency of "unfolded" wide binaries produced by this mechanism is too small even for the field, and much smaller than in young groups such as BPMG. 
Another strong prediction of the unfolding scenario is the high eccentricity of the outer orbits, the majority with $e>0.9$ for $a>10^{4}$ AU. There should be a correlation between the outer semimajor axis and the outer eccentricity. The eccentricities of very wide binaries can be determined statistically by accurate measurement of the relative motion (Tokovinin \& Kiyaeva 2016), soon to be available from Gaia. However, large incidence of subsystems implies the need to account for their motion, making such future study quite challenging.

The triple system $\alpha$ Cen A,B (HIP 71683 and 71681) and Proxima Cen (HIP 70890) is an excellent test case for the ejection scenario. Being the nearest, it should be typical of other similar systems (rather than exceptional). It is bound, given its age. The projected separation of Proxima is $s_{\text {proj }}=10.7 \mathrm{kAU}$, the total separation in space is $s=15 \mathrm{kAU}$, and the parabolic velocity is $V_{\text {par }}=0.51 \mathrm{~km} \mathrm{~s}^{-1}$. Wertheimer \& Laughlin (2006) calculated the binding energy of the wide pair $\mathrm{AB}, \mathrm{C}$ and found that it can be either positive or negative with roughly equal probability. The latest study by Kervella et al. (2017), however, shows that the triple system is bound and that the eccentricity of the outer orbit is moderate, 0.42 . Therefore this triple system has not been formed by unfolding. Interestingly, the outer and inner orbits are inclined by only $29^{\circ}$. The outer orbit thus has a large angular momentum and it is roughly aligned with the inner orbit.

\subsection{Cluster dissolution}

Moeckel \& Bate (2010) show that after 10 Myr of dynamical evolution of the dense simulated cluster, several wide binaries with $10^{4}<s<10^{5}$ AU are formed in its expanding halo. These binaries are wider than the original cluster size of $10^{4} \mathrm{AU}$, and half of them are triple or higher-order multiples. There were $15 \pm 7$ binaries with $s>10^{4} \mathrm{AU}$, or $1.7 \%$ for a total of 900 stars in that cluster. Kouwenhoven et al. (2010) estimate that, on average, one cluster produces one wide binary with $s>10^{4}$ AU. The frequency of such pairs in the field is $\sim 2 \%$. If all field wide pairs were formed by the cluster dispersal mechanism, a typical cluster should contain $\sim 50$ stars.

On the other hand, the frequency of pairs with $s>10^{4} \mathrm{AU}$ in the BPMG is $7 / 49=0.14 \pm 0.05$, significantly larger than in the field. Such a large fraction of wide pairs cannot be explained by either cluster dissolution or by the unfolding mechanisms. Joncour et al. (2017) reached the same conclusion regarding wide pairs in Taurus.

\subsection{Entrapment}

Makarov (2012) considered formation of very wide $(s \sim 1 \mathrm{pc})$ pairs from unrelated stars that are accidentally "entraped" into common motion in the Galactic potential. In principle, such pairs can survive for a long time. However, their motion is chaotic (not Keplerian) and they can dissolve as easily as they were formed. Makarov estimates that this mechanism is too inefficient for explaining wide pairs in the field. Moreover, wide binaries are more readily disrupted by passing stars or molecular clouds than by the smooth Galactic potential, so the large Jacoby radius of $\sim 1.8 \mathrm{pc}$ is not relevant for their survival (Weinberg et al. 1987).

\section{SUMMARY}

The separation distribution of stellar pairs in the low-density SFRs is nearly uniform (logarithmically) up to $10^{4} \mathrm{AU}$ and increases at larger separations. This upturn is associated with the initial fractal clustering of prestellar cores. It disappears progressively with increasing age, leaving a smoothly declining separation distribution in the field (Figure 3). Young moving groups such as BPMG (age $23 \mathrm{Myr}$ ) represent an intermediate case, with $14 \%$ of stars having companions wider than $10^{4} \mathrm{AU}$. This large fraction of wide pairs does not match such proposed formation mechanisms as cluster dissolution or unfolding of unstable triples. However, it can be explained easily as a remnant of the clustering, where a fraction of adjacent cores happen to be gravitationally bound. It is shown that the gas kinematics allows for a large enough fraction of bound core pairs if the motions are highly anisotropic and the resultant velocity difference between the cores is distributed almost normally, rather than by the Maxwell-Bolzmann law.

Stars in the field come from different environments. The frequency of wide pairs in the field therefore can indicate what fraction of the field stars were formed in the low-density SFRs like Taurus-Auriga and BPMG, and what fraction comes from larger and denser clusters (Patience et al. 2002; Reipurth et al. 2007).

\section{ACKNOWLEDGMENTS}

I appreciated the discussion of binary formation with B. Reipurth and P. Elliott. Referee's comments helped to improve this paper.

\section{REFERENCES}

Abt, H. \& Levy, S. 1976, ApJS, 30, 273

Alves, J., Lombardi, M. \& Lada, C. J. 2007, A\&A, 462, L17

André, P., Francesco, J. D., Ward-Thompson, D. et al., 2014, in Beuther, H., Klessen, R. S., Dullemond, C. P., \& Henning, T., eds, Protostars and Planets VI. Univ. Arizona Press, Tucson, AZ, p. 27

Bate, M. R., Clarke, C. J., \& McCaughrean, M. J. 1998, MNRAS; 297, 1163

Bate, M. R. 2014, MNRAS, 442, 285

Conneley, M. S., Reipurth, B., \& Tokunaga, A. 2008, AJ, 135, 2526

Duchêne, G. \& Kraus, A. 2013, ARAA, 51, 269 (ArXiv:1303:3028)

Duquennoy, A. \& Mayor, M. 1991, A\&A, 248, 485 (DM91)

Elliott, P. \& Bayo, A. 2016, MNRAS, 459, 4499

Goodwin, S. P., Kroupa, P., Goodmanm A., \& Burkert, A. 2007, in Protostars and Planets V. Eds. Reipurth, B., Jewitt, D., \& Keil, K. Univ. Arizona Press, Tucson, AZ, p. 133

Gomez, A. M., Hartmann, L., Kenyon, S. J. \& Hewett, R. 1993, AJ, 105, 1927

Heyer, M. Krawczyk, C., Duval, J. \& Jackson, J. M. 2009, ApJ, 699, 1092

Joncour, I., Duchêne, G. \& Moraux, E. 2017, A\&A, 599, 14

Kaczmarek, T., Olczak, C. \& Pfalzner, S. 2011, A\&A, 528, 144

Kervella, P., Thévenin, F. \& Lovis, C. 2017, A\&A, 598, L7

King, R. R., Goodwin, S. P., Parker, R. J., \& Patience, J. 2012, MNRAS, 427, 2636

Kounkel, M., Megeath, S. T., Poteet, C. A. et al. 2016, ApJ, 821, 52

Kouwenhoven, M. B. N., Goodwin, S. P., Parker, R. J. et al. 2010, MNRAS, 404, 1835

Kraus, A. L. \& Hillenbrand, L. A. 2008, ApJ, 686, L111

Kraus, A. L. \& Hillenbrand, L. A. 2009, ApJ, 703, 1511

Kraus, A. L., Ireland, M. J., Martinache, F. \& Hillenbrand, L. A. 2011, ApJ, 731,8

Larson, R. B. 1995, MNRAS, 272, 213

Larson, R. B. 2007, Rep. on Progr. in Phys., 70, 337 (ArXiv:0701.733)

Law, N. M., Dhital, S., Kraus, S. et al. 2010, ApJ, 720, 1727

Lomax, O., Whitworth, A. P., Hubber, D. A. et al. 2015, MNRAS, 447, 1550

Makarov, V. V., Zacharias, N. \& Hennessy, G. S. 2008, ApJ, 687, 566

Makarov, V. V. 2012, MNRAS, 421, L12 
Mamajek, E. E. \& Bell, C. P. M. 2014, MNRAS, 445, 2169

Moeckel, N. \& Bate, M. R. 2010, MNRAS, 404, 721

Patience, J., Ghez, A. M., Reid, I. N. \& Matthews, K. 2002, AJ, 123, 1570

Raghavan, D., McAlister, H. A., Henry, T. J. et al. 2010, ApJS, 190, 1

Reipurth, B., Guimarães, M. M., Conneley, M., \& Mally, J. 2007, AJ, 134, 2272

Reipurth, B. \& Mikkola, S. 2012, Nature, 492, 221

Reipurth, B., Boss, A. P., Clarke, C. J. et al. 2014 in Beuther, H., Klessen, R. S., Dullemond, C. P., \& Henning, T., eds, Protostars and Planets VI. Univ. Arizona Press, Tucson, AZ, p. 267

Simon, M. 1997, ApJ, 482, L81

Sterzik, M., Durisen, R. H., Zinnecker, H. 2003, A\&A, 411, 91

Tobin, J. J., Looney, L. W., Li, Z.-Y. et al. 2016, ApJ, 818, 73

Tokovinin, A. \& Lépine, S. 2012, AJ, 144, 102

Tokovinin, A. 2014, AJ, 147, 87

Tokovinin, A. \& Kiyaeva, O. 2016, MNRAS, 456, 2070

Weinberg, M.D., Shapiro, S.L., \& Wasserman, I. 1987, ApJ, 312, 367

Wertheimer, J. G. \& Laughlin, G., 2006, AJ, 132, 1995 